\title{
Clinical and Statistical Study on Canine Impaction
}

\author{
Coșarcă Adina-Simona1, Păcurar Mariana², Nagy-Bota Monica-Cristina³, Ormenișan Alina ${ }^{4}$ \\ 1 PhD student, Department of Oral and Maxillofacial Surgery, Faculty of Dental Medicine, University of Medicine and Pharmacy, Tîrgu Mureș, Romania \\ 2 Department of Pedodontics and Orthodontics, Faculty of Dental Medicine, University of Medicine and Pharmacy, Tîrgu Mureș, Romania \\ ${ }^{3} \mathrm{PhD}$ student, Department of Anatomy and Embryology, Faculty of Dental Medicine, University of Medicine and Pharmacy, Tîrgu Mureș, Romania \\ 4 Department of Oral and Maxillofacial Surgery, Faculty of Dental Medicine, University of Medicine and Pharmacy, Tîrgu Mureș, Romania
}

\begin{abstract}
Aim: The aim of this study was to perform a clinical and statistical research on permanent impacted canine patients among those with dental impaction referred to and treated at the Oral and Maxillo-Facial Surgery Clinic of Tîrgu Mureș, over a four years period (2009-2012).

Materials and methods: The study included 858 patients having dental impaction, and upon clinical records, different parameters, like frequency, gender, age, quadrant involvement, patient residence, associated complications, referring specialist and type of treatment, related to canine impaction, were assessed.

Results: The study revealed: about 10\% frequency of canine impaction among dental impactions; more frequent in women, in the first quadrant (tooth 13); most cases diagnosed between the age of 10-19 years; patients under 20 were referred by an orthodontist, those over 20 by a dentist; surgical exposure was more often performed than odontectomy.

Conclusions: Canine impaction is the second-most frequent dental impaction in dental arch after third molars; it occurs especially in women. Due to its important role, canine recovery within dental arch is a goal to be achieved, whenever possible. Therefore, diagnose and treatment of canine impaction requires an interdisciplinary approach (surgical and orthodontic).
\end{abstract}

Keywords: dental impaction, surgery, canine impaction

Received: 6 March 2013

\section{Introduction}

The canine plays an important role within the dento-maxilar complex: functionally, by enabling the canine guidance of mandible movement; esthetically, by being in the frontal region of the dental arch, and by supporting canine bosa and facial aspect; prosthetically, by having the longest root; and by being the most longevive tooth.

Dental impaction (dental inclusion) is defined as the intraosseous retention of a completely developed tooth, or as the failure of tooth eruption in its appropriate site, within the dental arch, during its normal period of eruption (deep impaction) [1]. Some authors use the term dental enclosure for teeth that are partially boneless and located below the gingival fibromucosa [2].

Canine impaction occurs often, being the second-most frequent impacted tooth in the dental arch after the third molars [3].

Canine impaction may be unilateral or bilateral. The canine can remain impacted on the bucal or on the oral (palatinal/lingual) site of the dento-alveolar arch, or centrally (intermediate level between the two bone plates). Canine impaction in the edentulous maxilla or mandible represents a separate entity.

Since the canine plays an active part within the dentoalveolar arch, over the entire life, the treatment of canine impaction should attempt its recuperation whenever it is possible. Therefore, often, an interdisciplinary protocol

Correspondence to: Adina-Simona Coșarcă

E-mail: adicosarca@yahoo.com (orthodontical and surgical) should be followed in order to recover and morphologically and functionally integrate the canine. Thus, treatment can be: conservative, either orthodontical (by creating space within the dental arch in order to gain spontaneous eruption), or combined (orthodontical and surgical) - first, the orthodontist creates a sufficient eruption space within the dental arch, then, the oral surgeon exposes surgically the impacted canine and places an orthodontic device (bracket) upon the canine in order to help its eruption; or, it can be radical - odontectomy (removal of the impacted canine), when recovery cannot be achieved.

\section{Materials and methods}

A clinical and statistical study on canine impaction was performed in patients exhibiting dental impaction, that were referred and treated at the Oral and Maxillo-Facial Surgery Clinic of Tîrgu Mureș in the 2009-2012 period, investigating several different parameters.

The study included 858 cases of dental impaction, the patients being aged between 10 and 80 years.

The lack of a permanent canine within the dental arch, proper clinical records, radiological (X-ray) and laboratory investigations, and adequate surgical protocols were considered mandatory criteria in order to perform this study. Based on these criteria, multiple parameters concerning canine impaction were studied, such as the age and gender of patients, provenience (urban vs. rural), frequency or quadrant involvement. X-rays were used to investigate the relationship of the impacted canine with neighboring teeth, 
Table I. Frequency of impaction with a $95 \%$ confidence interval

\begin{tabular}{lccc}
\hline & Frequency & Percentage & Confidence interval \\
\hline Right upper canine (13) & & & \\
$\quad$ Females & 34 & $65.4 \%$ & $50.9-78 \%$ \\
$\quad$ Males & 18 & $34.6 \%$ & $22.0-49.1 \%$ \\
$\quad$ Total & 52 & $100.0 \%$ & \\
Left upper canine (23) & & & \\
$\quad$ Females & 21 & $60 \%$ & $42.1-76.1 \%$ \\
$\quad$ Males & 14 & $40 \%$ & $23.9-57.9 \%$ \\
$\quad$ Total & 35 & $100.0 \%$ & \\
Left lower canine (33) & & & \\
$\quad$ Females & 2 & $66.7 \%$ & $9.4-99.2 \%$ \\
$\quad$ Males & 1 & $33.3 \%$ & $0.8-90.6 \%$ \\
Total & 3 & $100.0 \%$ & \\
\hline
\end{tabular}

maxillary sinus, mandibular canal. The presence of associated complications, such as infections (abscesses spread towards one of bone plates - bucal or palatinal/lingual), likely obstacles interfering with canine eruption (temporary canine persistence, odontomas, and supernumerary teeth), or cleft lip and palate, were also noted.

Furthermore, by reviewing clinical records, we were able to establish whether the patient was referred by a specialist (orthodontist, dentist, general physician), or presented spontaneously. Finally, we analyzed the surgical protocols and the type of treatment applied to each patient (combined surgical-orthodontic or odontectomy).

Data were collected into a Microsoft Excel file and processed with the Epi Info system.

\section{Results}

Regarding the frequency of canine impaction, the study showed 91 cases $(10.60 \%)$ from the total of 858 cases exhibiting dental impaction.

Considering maxillary tooth impaction, the study revealed: on the first quadrant, tooth $13-52$ cases $(6.1 \%)$; tooth $14-2$ cases $(0.2 \%)$; tooth $15-2$ cases $(0.2 \%)$; tooth $18-133$ patients $(15.5 \%)$; on the second quadrant, tooth $23-35$ cases (4.1\%); tooth $24-2$ cases $(0.2 \%)$; tooth 25 -1 cases $(0.1 \%)$; tooth $28-107$ cases $(12.5 \%)$.

Considering mandibular tooth impaction, the study revealed: on the third quadrant, tooth $33-3$ cases $(0.3 \%)$;

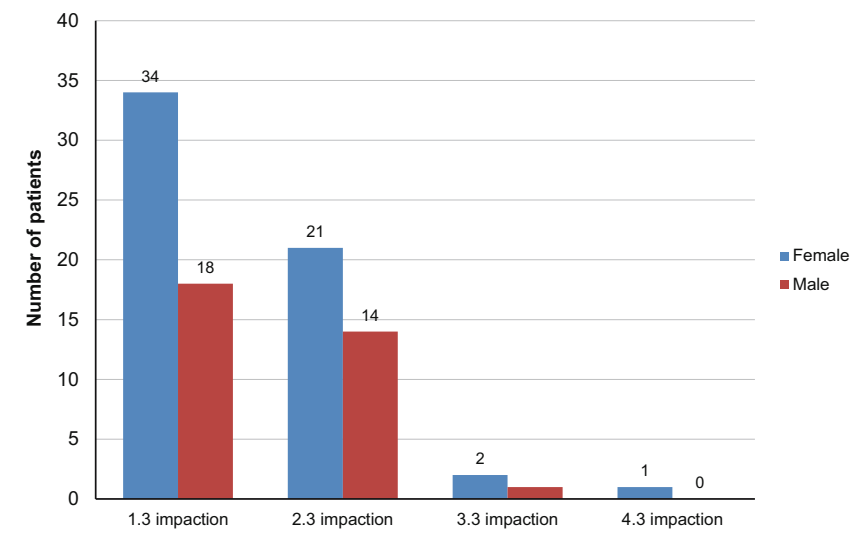

Fig. 1. Gender distribution of canine impaction
Table II. The type of treatment

\begin{tabular}{lccc}
\hline & Frequency & Percentage & Confidence interval \\
\hline Right upper canine (13) & & & \\
$\quad$ Exposure & 28 & $53.8 \%$ & $39.5 \%-67.8 \%$ \\
$\quad$ Odontectomy & 24 & $46.2 \%$ & $32.2 \%-60.5 \%$ \\
$\quad$ Total & 52 & $100.0 \%$ & \\
Left upper canine (23) & & & \\
$\quad$ Exposure & 17 & $48.6 \%$ & $31.4 \%-66.0 \%$ \\
$\quad$ Odontectomy & 18 & $51.4 \%$ & $34.0 \%-68.6 \%$ \\
$\quad$ Total & 35 & $100.0 \%$ & \\
Left lower canine (33) & & & \\
$\quad$ Exposure & 2 & $66.7 \%$ & $9.4 \%-99.2 \%$ \\
$\quad$ Odontectomy & 1 & $33.3 \%$ & $0.8 \%-90.6 \%$ \\
$\quad$ Total & 3 & $100.0 \%$ & \\
Right lower canine (34) & 1 & $100 \%$ & \\
$\quad$ Odontectomy & 1 & $100 \%$ & \\
\hline & & & \\
\hline
\end{tabular}

tooth $34-1$ cases $(0.1 \%)$; tooth $35-0$ cases $(0.0 \%)$; tooth $38-337$ cases (39.3\%); on the fourth quadrant, tooth 43 -1 cases $(0.1 \%)$; tooth $44-2$ cases $(0.2 \%)$; tooth $45-0$ cases $(0.0 \%)$; tooth $48-335$ cases $(39.0 \%)$.

Canine impaction was found in 58 cases $(63.73 \%)$ in female patients, and in 33 cases $(36.26 \%)$ in male patients (Figure1).

Regarding age, most cases were diagnosed between 10 and 19 years $(38.5 \%$ tooth $13 ; 42.9 \%$ tooth $23 ; 66.7 \%$ tooth 33), and between 20 and 29 years ( $25.0 \%$ tooth 13 ; $20.0 \%$ tooth 23) (Figure 2).

Regarding the distribution on dental arches, canine impaction was found more frequent in the maxilla (95.60\%), especially on the right side, tooth 13 (57.14\%). The only case found in right mandible, at tooth 43 , was considered irrelevant and it was excluded from the subsequent calculations (Table I).

Regarding quadrants involvement by canine impaction, our findings were: first quadrant - 52 cases (57.14\%), second quadrant -35 cases $(38.46 \%)$, third quadrant -3 cases $(3.92 \%)$, fourth quadrant -1 case $(1.09 \%)$ (Table I).

With respect to the area of residence, 56 cases $(61.53 \%)$ came from urban area -33 cases $(63.5 \%)$ tooth $13 ; 21$ cases (60.0\%) tooth 23 ; and 2 cases (66.7\%) tooth 33 ; and 34 cases $(37.36 \%)$ came from a rural area -19 cases $(36.5 \%)$ tooth 13 ; 14 cases $(40.0 \%)$ tooth 23 ; 1 case $(33.3 \%)$ tooth 33 .

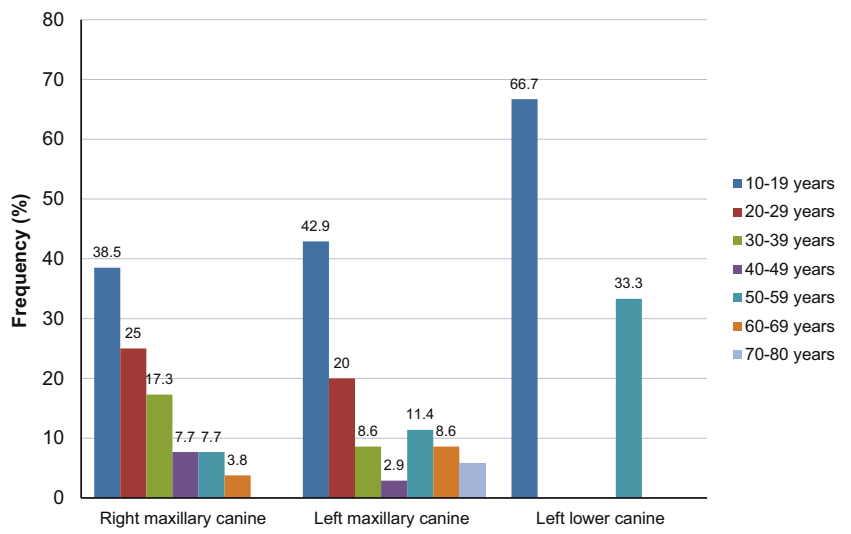

Fig. 2. The frequency of canine impaction by age groups 
Regarding specific features of canine impaction, the study showed: close relation to the maxillary sinus, in one patient $(1.09 \%)$ - complaining of neuralgic pain on $\mathrm{V} / 2$ right nerve, and requiring odontectomy; close relation to the mandibular canal, one case $(1.09 \%)$, tooth 43; association of infectious complications (abscesses) 12 cases $(13.18 \%)$ bucal and 5 cases $(5.49 \%)$ palatinal; temporary canine persistence, 23 cases $(25.27 \%)$; associated odontomas, 9 cases $(9.89 \%)$, and supernumerary teeth, 5 cases $(5.49 \%)$, all precluding permanent canine eruption; association of operated cleft lip and palate, 3 patients $(3.26 \%)$ - surgical exposure and bracketing being performed.

Concerning the specialist who referred patient to oral surgeon, the study revealed: all patients under 20 years (37 cases, $40.65 \%$ ) were referred by an orthodontist; most of the patients over 20 years were referred by a dentist (canine impaction being discovered accidentally during routine dental treatment or X-ray), 20 cases $(21.97 \%)$ were referred by a general physician, and 3 patients $(3.29 \%$ presented spontaneously.

Regarding the applied treatment (surgical-orthodontic recovery or surgical removal) the study revealed relatively close values of surgical exposure ( 47 cases, $51.60 \%$ ) and odontectomy ( 44 cases, $48.35 \%$ ), as presented in Table II.

\section{Discussion}

As far as the frequency of canine impaction is concerned, our study indicates that canine impaction follows as frequency the third molar, besides other studies, performed by Güzduz in 2011 [4], and Fardi in 2011[5], where the maxillary canine was found to be the most frequently impacted tooth.

Concerning the patients' gender, our study reveals the predominance of canine impaction in women $(63.73 \%)$, similarly to a study conducted by an orthodontist in 2012 (female:male ratio 2:1) [6].

Our study showed that canine impaction usually is diagnosed between the ages of 10-19 years, unlike the aforementioned study, which found that canine impaction was diagnosed mostly between $13-15$ years [6]. Also, our study included patients over 40 years of age, unlike the study mentioned above, where dental impaction was diagnosed mostly under the age of 37 .

Referring to maxilla/mandible and quadrants involvement, in our study canine impaction was more frequent in the first quadrant, on the right side of the upper dental arch, while some studies report more cases on the left side $[6,7]$.

According to our study, canine impaction was significantly more frequent in patients coming from urban areas, this being consistent with data from literature $[2,3,7]$, although there is some controversy regarding this aspect. A probable explanation might be represented by the fact that these patients visit their doctors more often.

Canine impaction was associated with neuralgia and abscesses in our study, and we found temporary canine persistence in $25.27 \%$ of cases, unlike the study conducted in 2012 in Sibiu, where this percentage was 62\% [6].

Usually, patients exhibiting canine impaction are diagnosed by the orthodontist during childhood or early adulthood. As patients age, their dentist or general physician refer them to the oral and maxillofacial surgeon.

The treatment of choice in canine impaction is canine recovery (orthodontical or combined surgical-orthodontical), whenever is possible, otherwise, when complications are associated, usually surgical removal is mandatory.

Impacted mandibular canines are rarely found in our clinic. A study carried out in Turkey found that impacted mandibular canines came after maxillary impacted canines, but, compared to our study, the author revealed an increase of the number of impacted mandibular canines (65 patients in 8 years, compared to our study, where we found 4 cases in 4 years) [8].

\section{Conclusions}

1. Canine impaction is the second-most frequent impacted tooth within the dental arch, after the third molars.

2. Canine impaction occurs more frequently in women.

3. The most frequent canine involved in impaction is the right maxillary canine.

4. The treatment of canine impaction is complex, relative to its morphological and functional recovery within dental arch.

5. The therapy of canine inclusion requires an accurate diagnosis and interdisciplinary approach based on the collaboration and careful coordination between the surgeon and orthodontist, to diagnose and determine the optimal therapeutic strategy, and to inform patients properly about the consequences of impacted canine in adulthood (possible associated complications).

\section{References}

1. Ionescu E. Anomaliile dentare. Editura Universitară, București, 2005.

2. Timoșca G, Burlibașa C. Chirurgie buco-maxilofacială, Editura Didactică și Pedagogică, București, 1983

3. Burlibașa C. Chirurgie orală și maxilo facială. Editura Medicală, București, 2001, 259-263.

4. Günduz K, Acikgöz A, Egrioglu E. Radiologic investigation of prevalence, associated pathologies and dental anomalies of non-third molar impacted teeth in Turkish oral patients. Chin. J Dent Res. 2011;14(2):141-6.

5. Fardi A, Kondilidon-Sidira A, Bachour Z, Parisis N, Tsirlis A. Incidence of impacted and supranumerary teeth-a radiografic study in a North Greek population. Med. Oral. Patol. Oral. Cir. Bucal. 2011;16(1):55-61.

6. Smarandache A, Palada I, Sabău M. Studiu statistic al incluziei de canin maxilar. Acta Medica Transilvanica. 2012;2(4):129-132.

7. Bucur A. Compendiu de chirurgie oro-maxilo-facială. Q Med Publishing. 2009;:1:115-116.

8. Yavus MS, Aras MH, Büyükkurt MC, Tozoglu S. Impacted mandibular canines. J. Contemp. Dent. Pract. 2007;8(7):78-85. 